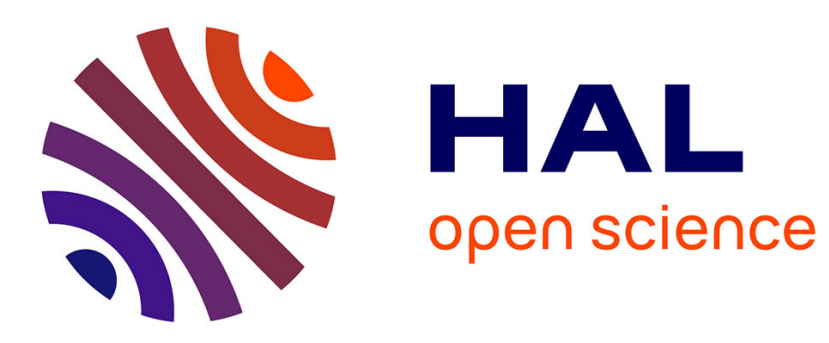

\title{
Phenotypic plasticity through disposable genetic adaptation in ciliates
}

Rik Verdonck, Delphine Legrand, Staffan Jacob, Hervé Philippe

\section{To cite this version:}

Rik Verdonck, Delphine Legrand, Staffan Jacob, Hervé Philippe. Phenotypic plasticity through disposable genetic adaptation in ciliates. Trends in Microbiology, In press, 10.1016/j.tim.2021.06.007 . hal-03433044

\section{HAL Id: hal-03433044 \\ https://hal.science/hal-03433044}

Submitted on 29 Nov 2021

HAL is a multi-disciplinary open access archive for the deposit and dissemination of scientific research documents, whether they are published or not. The documents may come from teaching and research institutions in France or abroad, or from public or private research centers.
L'archive ouverte pluridisciplinaire HAL, est destinée au dépôt et à la diffusion de documents scientifiques de niveau recherche, publiés ou non, émanant des établissements d'enseignement et de recherche français ou étrangers, des laboratoires publics ou privés. 


\title{
Phenotypic plasticity through disposable genetic adaptation in ciliates
}

\author{
Rik Verdonck ${ }^{1,2}$, Delphine Legrand ${ }^{1}$, Staffan Jacob $^{1}$, Hervé Philippe $^{1}$ \\ ${ }^{1}$ Station d'Écologie Théorique et Expérimentale, UPR 5321, CNRS, Moulis, France \\ ${ }^{2}$ Current address: Research group Environmental Biology, UHasselt, Belgium \\ Corresponding author: Rik Verdonck (rik.verdonck@gmail.com)
}

\begin{abstract}
Ciliates possess an extraordinary genetic system in which each cell harbours two distinct kinds of nucleus, a transcriptionally active somatic nucleus and a quiescent germline nucleus. The latter undergoes classical, heritable genetic adaptation, while adaptation of the somatic nucleus is only short-term and thus disposable. The ecological and evolutionary relevance of this nuclear dimorphism have never been well formalized, which is surprising, given the long history of using ciliates such as Tetrahymena and Paramecium as model organisms. We present a novel, alternative explanation for ciliate nuclear dimorphism, which we argue should be considered an instrument of phenotypic plasticity by somatic selection on the level of the ciliate clone, as if it were a diffuse multicellular organism. This viewpoint helps to put some enigmatic aspects of ciliate biology into perspective and presents the diversity of ciliates as a large natural experiment that we can exploit to study phenotypic plasticity and organismality.
\end{abstract}

\section{Keywords}

Ciliates, somatic selection, Weismann barrier, organismality, ploidy, intergenomic conflict, phenotypic plasticity

\section{Highlights}

- Somatic selection, a form of phenotypic plasticity that was hitherto only described in multicellulars, is also present in ciliates.

- Ciliates have evolved two genomes, one of which is a carrier of short-term disposable adaptations, serving the long-term survival of the other. 
- Natural history of ciliates and some of their genomic specificities need to be reconsidered in the light of phenotypic plasticity by somatic selection.

\section{The extraordinary genetic system of ciliates}

Ciliates are a ubiquitous eukaryotic life form present in most aquatic ecosystems. Most ciliates (e.g., Tetrahymena, Oxytricha and Paramecium) take a central place in food webs as planktonic predators of microorganisms [1]. Some species are (facultatively) sessile, commensal or parasitic. Ciliates have a unique genetic system that is, despite their merits as model organisms, relatively poorly understood. They exhibit nuclear dimorphism (see glossary): each cell harbours two different kinds of nuclei. The cell's phenotype is determined by the expression of a somatic nucleus (macronucleus). Meanwhile, the germline nucleus (micronucleus) is quiescent. Being facultatively sexual, periods of asexual reproduction, during which both nuclei divide independently, are alternated with sexual reproduction, during which meiotic products of the micronucleus are exchanged between mother cells (see fig. 2 in [2]). Upon fertilization, these gamete nuclei fuse to form a new nucleus, which divides and gives rise to both a new micro-and macronucleus. Next, the old macronucleus disappears.

Since a genetic change has a strikingly different fate depending on whether it occurs in the micro- or macronucleus, the two nuclei have distinct evolutionary dynamics. Fig. 1 illustrates how in ciliates, diversity is generated and selected from at the level of the macronuclei, which have an extraordinary capacity to generate somatic genetic diversity (see below). Since the macronucleus determines the phenotype, it is under selection during periods of vegetative growth, adapting to its environment the same way the genome of any other asexual unicellular organism does. Meanwhile, the silent micronucleus independently accumulates mutations that only become exposed to selection upon sexual reproduction, when a new macronucleus is derived from the new micronucleus. This leads to the presence of two levels of selection: the individual cell with its phenotype (and hence its fitness) determined by its macronucleus, and the entire clone with its fitness determined by the ability of its micronucleus to generate well-adapted macronuclei.

The only nucleus that can ultimately contribute to the gene pool is the micronucleus, which therefore constitutes the genotype of the clone. We argue that on an evolutionary level, the clone can be considered the main unit of selection [3], and hence should be considered a genet (genetic [4] or evolutionary individual [5]). The ciliate cell can be thought of as a ramet (functional [4] or physiological individual [5]). In most ciliate species, cells separate after asexual division. The typical ciliate clone may thus be viewed as a diffuse multicellular organism (Box 1) consisting of physiologically independent somatic cells that share a germline.

\section{Variable environments: phenotypic plasticity and bet-hedging}

Here, we propose that the adaptation of the macronucleus constitutes a form of phenotypic plasticity: the ability of a single genotype to give rise to multiple phenotypes in different environments [6-8]. Phenotypic plasticity takes contrasting forms including passive phenotypic changes, generalized responses or developmental switches $[9,10]$. Examples of such sensing and responding strategies in microorganisms include the lactose operon in Escherichia coli [11] or fruiting body formation from 
individual Dictyostelium cells upon starvation [12]. By modulating the direct link between genotype and phenotype, phenotypic plasticity is key to an organism's fitness [13]. When phenotypic plasticity provides a fitness benefit sufficiently high to select for its genetic basis and when several environments are regularly encountered during evolutionary history, it can improve the match between phenotype and environment and is, therefore, adaptive [14].

However, cases exist where reliable cues to predict the future state of the environment are unavailable. Such conditions are expected to favour diversified bet-hedging strategies, where an individual increases the phenotypic variation among its offspring (be it sexual or asexual), resulting in high fitness of some offspring in a particular environment, while others may perform poorly. In this manner, the temporal variation in fitness of the genotype is buffered [15-17]. Various molecular mechanisms exist to constitutively generate phenotypic heterogeneity for a given genotype, allowing organisms to persist in fluctuating environments [18]. Well-characterized examples of bet-hedging include persisters in bacteria [19], and viral latency [20]. A bet-hedging strategy is not exclusive and heterogeneity can be fine-tuned by phenotypic plasticity, such as in amphicarpic annual seeds [21].

Another strategy to deal with unpredictable environmental fluctuations is somatic selection $[8,10]$. This is a non-canonical form of phenotypic plasticity where the phenotype-environment match relies on the production of a wide range of phenotypic states within one individual, out of which the best performing variants are selected through feedback between the environment and the developing individual $[8,22]$. Examples of somatic selection include the development of plant roots as a function of their ability to provide the plant with nutrients and water [22], or human learning, where among trillions of synaptic connections, some are eliminated while others are reinforced upon their successful interaction with environmental cues [23]. Mechanistically, somatic selection does not look like a typical form of phenotypic plasticity with a canonical phenotype-to-environment mapping (reaction norm) shaped by evolutionary history. Conversely, in somatic selection, the feedback between environment and phenotype takes place within the lifetime of the individual [10].

Both in somatic selection and diversified bet-hedging, multiple phenotypic variants are generated from one genotype, but in bet-hedging, the distribution of initially generated variants is generally considered independent of the current environment (but see [21]), while in somatic selection, the distribution of phenotypic variants is modulated through constant feedback between phenotype and environment [10]. In addition, bet-hedging and plasticity by somatic selection differ on the level at which selection is acting. In somatic selection, variability is generated and selected from within an individual, while bet-hedging is based on inter-individual phenotypic variation among offspring. The distinction is generally clear for organisms with classical life cycles. In plants, variation in seed dormancy is a form of diversified bet-hedging [17] while root proliferation or atrophy as a function of environmental conditions is a form of phenotypic plasticity by somatic selection [22]. Conversely, in strictly clonal microbes, the distinction may be dubious. Phenotypic heterogeneity within a clone can be considered diversified bet-hedging [16], but if members of a clone are joined together as a unit of selection it may also be seen as part of a somatic selection strategy.

\section{Disposable genomes allow for somatic selection}

To date, phenotypic plasticity by somatic selection has only been conceptualized for multicellular organisms: diversity is generated and selected from at the level of somatic tissues, while the presence of 
a physically distinct germline guarantees genetic continuity and a return to the naive state at every new generation. Since the ciliate clone is an evolutionary individual with its genotype contained in its germline micronuclei, genetic adaptation of its macronuclei is disposable and merely serves to generate the best vehicles to escort its genotype to the next sexual generation, as does the soma of multicellulars. We therefore argue that the disposable genetic adaptation of the ciliate macronucleus may be seen as an overlooked case of somatic selection, occuring at the level of a clone of unicellulars.

A comparison with the vertebrate adaptive immune system, a prime example of phenotypic plasticity by somatic selection, is illustrative (Fig. 2). In the same way that the combination of all antigen-recognizing cells constitutes the immunological phenotype of a vertebrate individual, the total phenotype of the ciliate clone results from the ensemble of single-cell phenotypes that have been selected by the environment. The phenotypic plasticity of the clone resulting from somatic selection is distinct from, but complementary to the more classical phenotypic plasticity at the level of the physiological individual (the cell).

The parallel with multicellulars (Box 1 ) is not absolute. In both cases, the expression of somatic genomes produces heterogeneous phenotypes, on which selection can operate depending on individual cell performance. The resulting repertoire of somatic genomes is associated with a unique germline that evolves at the time scale of sexual generations. However, in ciliates, each somatic genome is associated with its own physical copy of the germline while in multicellulars all somatic genomes are associated with a single, physically distinct germline. In a multicellular, selection acts more homogeneously on all physically coupled somatic genomes, whereas cells of a ciliate clone move independently and can disperse away from each other (Fig. 1). As a result, somatic genomes of a ciliate clone can simultaneously be exposed to different selective pressures while silent mutations in their physically associated germline genomes hitchhike along.

\section{Amitosis generates a huge somatic genomic variability}

Phenotypic plasticity by somatic selection in ciliates is facilitated by several unusual properties of the macronucleus, directed towards the generation of somatic genetic heterogeneity. While the micronucleus is a typical diploid eukaryotic nucleus that undergoes mitosis during phases of asexual reproduction, the macronucleus is polyploid and divides amitotically. The exact process of macronucleus formation from the micronucleus template differs markedly between ciliate lineages, but always involves replication to a higher ploidy, fragmentation into smaller chromosomes, and sometimes extensive shuffling $[2,24,25]$. In Tetrahymena thermophila, the micronucleus has five diploid chromosomes, while the macronucleus has 181 chromosomes in about 45 copies each. In Oxytricha trifallax, macronuclear chromosomes are fragmented down more or less to the level of individual genes, giving rise to 16,000 chromosomes occurring in copy numbers of up to several thousand each.

Along with centromeres, the macronucleus has lost its ability to form the mitotic spindle and metaphase plate, structures that normally guarantee the equal distribution of sister chromatids to the daughter nuclei. Therefore, the macronucleus divides amitotically, with a stochastic component to the partitioning of chromosomes between daughter nuclei, generating substantial somatic genetic diversity (Fig. 3). 
First, amitosis impacts the level of heterozygosity in the macronucleus. In a diploid multicellular, any locus that is heterozygous in the germline will be heterozygous in all somatic genomes, regardless of the number of divisions. Conversely, the partitioning of parental alleles during macronuclear division is a stochastic process. In any given macronuclear lineage and in the absence of selection, each parental allele, as well as any de novo mutation, tends to either get lost or drift to fixation (Fig. 3, proportion of colour shades), a phenomenon known as phenotypic assortment [26]. In laboratory experiments, phenotypic assortment has long been used to obtain populations fully homozygous for a chromosome carrying a selective marker (e.g., antibiotic resistance). Fixation by neutral drift is expected to occur after $\sim 200$ divisions when ploidy is $\sim 45$ as in $T$. thermophila [26]. In species with higher ploidy, such as Paramecium tetraurelia (ploidy $\sim 800$ ) or 0 . trifallax (ploidy $\sim 2000$ ), fixation may occur much later, or may not happen within the lifetime of the clone. The fixation of an allele may be accelerated by directional selection, or it may be prevented by balancing selection. The result of phenotypic assortment is the production of a multitude of combinations of allele proportions at different loci, among the cells of a clone.

Second, amitosis has an impact on the ploidy of macronuclear chromosomes. While during amitosis, daughter macronuclei may inherit about equal total numbers of chromosomes, the partitioning of each individual chromosome may vary by chance $[27,28]$. This leads to stochastic copy number variations of individual macronuclear chromosomes among cells (Fig. 3, height of bars), on which selection can act. The degree of stochasticity in this system may vary between species and is subject of debate. Some studies suggest that regulatory mechanisms prevent lethal ploidy variations [27,29], while others suggest that balancing selection is sufficient $[28,30]$. Partial ploidy changes are a quick and reversible way to alter patterns of gene expression. In Paramecium bursaria copy number variation of macronuclear chromosomes is low for chromosomes harbouring housekeeping genes and high for those carrying environmental response genes [31]. Likewise, in the presence of toxic $\mathrm{Cd}^{2+}$, the copy number of a chromosome harbouring two metallothionein genes increased five-fold in $T$. thermophila [32]. These partial ploidy changes were reversible upon relief from heavy metal stress, and could be re-induced upon a new exposure to $\mathrm{Cd}^{2+}$ in as little as a week, an interesting example of reversible phenotypic plasticity. While partial ploidy changes and phenotypic assortment certainly act in the wild, it remains to be evaluated to what extent they contribute to phenotypic plasticity by somatic selection.

\section{Ciliate somatic selection and intergenomic conflict}

Evolutionary conflict arises when multiple genetic entities present within an individual have divergent interests, where they would benefit from moving the phenotype in opposite directions [33,34]. Conflict can occur between genetic entities within the same genome (intragenomic conflict [35]), between multiple genomes within an organism (intergenomic conflict, e.g., [36]), between soma and germline within a multicellular organism (cancer [37]) or even between reproductive and non-reproductive casts within a eusocial insect colony [38]. Multiple solutions appeared to reduce these costly conflicts, for instance, uniparental inheritance of mitochondria [39], cell cycle checkpoints to prevent neoplastic growth [37], or policing worker-laid eggs in honeybees [38]. 
Within a ciliate clone, the presence of multiple levels of selection may lead to divergent interests between micro- and macronucleus [40,41]. From the perspective of the micronucleus, selection of the best macronuclear genomes within the clone provides the best odds for survival until the next sexual reproduction. However, since sex leads to an evolutionary dead-end for the macronucleus, the latter may gain a short-term benefit from the loss of the micronucleus, especially if such lineage has a growth advantage because it no longer has to replicate an extra nucleus. This tension sets the stage for intergenomic conflict that bears similarities with conflict between genomes of a cancer cell and the germline (selection on the somatic cell level $[34,37,42]$ ), or between nuclei in multinucleate ascomycete fungi due to within-mycelium selection [43]. The question is how this potential conflict is resolved in ciliates.

One extreme resolution is the complete "victory" of the soma through the loss of the micronucleus, as seen in some Tetrahymena species [44]. Inevitably, such lineage also loses sexual recombination, and it is unclear whether the primary driver of micronucleus loss is the resolution of intergenomic conflict, or other dynamics related to the loss of sex. At a macroevolutionary level, the long-term survival of asexual lineages is generally limited to stable environments [45] and ciliates are no exception: although amicronucleated lineages in laboratory cultures have been known for long (e.g., [46]), in nature, they have only been described in the genus Tetrahymena [44].

Conversely, amacronucleated lineages have only been observed after experimental manipulation and are unstable. Nonetheless, the ability to divide the macronucleus was lost in the class Karyorelictea, where a new macronucleus is generated from the micronucleus at each cell division $[24,47]$. Whether this loss evolved primarily as a resolution of genomic conflict, or rather as a loss of plasticity, needs further research. The long-term maintenance of phenotypic plasticity by somatic selection relies on the balance between the costs to produce numerous potentially unfit variants, and the benefit of the fit between the selected phenotypes and the encountered environments [22]. Perhaps in the case of Karyorelictea, this cost-benefit balance has tilted because of the stability of their environment (sediment), or their slower pace of life (millimetric cells).

The loss of the micronucleus, and perhaps also the loss of amitosis, are extreme resolutions of intergenomic conflict. The primary solution that allows the long-term coexistence of the two genomes lies in the presence of a Weismann barrier: a physical distinction between the soma and the germline. The Weismann barrier has been suggested to be an important mediator of conflict reduction in major evolutionary transitions, because it curtails the evolutionary potential of the lower units of selection, taking away their evolutionary interest to behave selfishly [48-50]. In multicellular organisms, the Weismann barrier is achieved through the confinement of the germline genome in specific cells. In ciliates, germline and somatic genomes are in close proximity within the same cell, so specific mechanisms should have evolved to prevent DNA exchanges. For instance, nuclear membranes never disappear throughout the ciliate life cycle [51], providing a simple physical barrier between micronucleus and macronucleus. Also, massive RNA-guided rearrangements occurring during micronucleus-to-macronucleus transformation effectively reduce the similarity between the two genomes, making homologous recombination less likely. In T. thermophila, about one-third of the micronucleus is excised under the form of Internal Eliminated Sequences (IES) that do not make it into the macronucleus. In O. trifallax, about $90 \%$ of the germline DNA is eliminated, and more than 3,500 genes are scrambled by inversion and permutation [52]. DNA elimination is generally viewed as a defence against transposons [41,53-55], and gene scrambling and imprecise excision have been discussed in the context of the generation of somatic diversity $[41,56]$. We hypothesise that these 
rearrangements may also have evolved to reinforce the ciliate Weismann barrier. The selective advantage of a single IES would be small for any of the proposed evolutionary drivers, so conceivably, the system emerged as a propensity of the proto-macronucleus to purge an abundant class of transposons, and was fine-tuned afterwards.

Even if the transfer of genetic material from the ciliate macronucleus back to the micronucleus seems impossible [57], the soma can transfer information to the next sexual generation. In animals, the Weismann barrier can be permeable for non-genetic information, and many traits show some degree of non-genetic heritability [58,59]. Examples include epigenetic markings on gamete genomes and the transfer of maternal hormones or antibodies through the placenta or egg yolk (Fig. 2). Likewise, the ciliate Weismann barrier is permeable for epigenetic information. During the remodelling of nuclei after sexual reproduction, the chromosome copy numbers in the new macronucleus in Stylonychia lemnae [60] and 0 . trifallax [61] are epigenetically regulated by RNA-guides that originate from the old macronucleus. In T. thermophila, a change in the structure of the macronucleus can become heritable in a non-Mendelian way: germline-limited sequences that were artificially introduced in the macronucleus were observed to persist in the newly generated macronucleus [62]. The importance of such transgenerational phenotypic plasticity remains to be determined, but it might provide a "peaceful" solution to the potential micronucleus/macronucleus conflict, i.e., an evolutionary compromise between the investment in phenotypic plasticity by somatic selection and in germline adaptation.

\section{Concluding remarks}

Ciliates are unlike any other unicellular eukaryote. We argue that they present a form of phenotypic plasticity so far only known in multicellular organisms, based on mechanisms otherwise seen in genetic adaptation. We propose that the framework of phenotypic plasticity by somatic selection (Fig. 1 and 2) within a diffuse multicellular organism (Box 1 ) helps to explain enigmatic ciliate properties such as amitotic nuclear division, the presence of a juvenile and senescent stage, or the intracellular Weismann barrier. Within a single ciliate cell, a mutation can lead to phenotypic plasticity or to genetic adaptation depending on which nucleus it happens in, possibly opening a grey zone between two concepts that are usually separated: plasticity and adaptation. This resonates with recent suggestions that organismality may be context-dependent [63], and with ongoing research about the implementation of the Weismann barrier and the evolutionary relevance of somatic genetic variation [64-68]. For such subjects, ciliates could provide a choice experimental model system for hypothesis testing. We hope that the viewpoint that we have put forward here will open stimulating debates on the evolutionary origin of multinucleated cells, the significance of cell-cell interactions and organismality.

\section{Acknowledgements}

We warmly thank Mike Singer, Darron Cullen, Emilie Snell-Rood and two anonymous reviewers for their precious advice. This work was supported by the French Laboratory of Excellence project entitled "TULIP" (ANR-10-LABX- 41), including a senior package attributed to HP (ANR-11-IDEX-0002-02). DL and SJ acknowledge financial support from the Agence Nationale de la Recherche for the projects POLLUCLIM (ANR-19-CE02-0021-01) and CHOOSE (ANR-19-CE02-0016) respectively. 


\section{Outstanding Questions:}

1. Are ciliates the only unicellular organisms capable of somatic selection?

2. What are the extent and evolutionary importance of the transgenerational transfer of traits acquired by the macronucleus through somatic selection? Transfer of information between sexual generations has been observed, but our knowledge on the communication between nuclei is fragmentary and limited to only a few species. A specialized form of genetic assimilation in which genetic novelties can do a test run in the macronucleus before being acquired by the micronucleus would be a spectacular example of Lamarckian-like adaptation.

3. Does somatic selection shed light on the evolutionary origin of nuclear dimorphism?

4. What about the third genome? The mitochondrial genome is under constant selection but without a reset at each sexual generation. This should result in interesting dynamics between short-term co-adaptation with the macronucleus, and continuity with the micronucleus.

5. What are the evolutionary consequences for an organism to harbour a copy of the germline in every cell? Formalizations about the population genetic consequences of having a "distributed germline" are lacking. It will be important to reconsider concepts such as inclusive fitness, cooperation and conflict in the context of the organismality of the ciliate clone.

6. How is the level of macronuclear diversity modulated? In multicellular examples of somatic selection, somatic diversity is under physiological control in order to balance out the costs and benefits of diversity (e.g., only a very specific region of the B lymphocyte genome is rearranged). 


\section{Glossary}

Amitosis: division of a cell nucleus without the mitotic mechanisms that normally ensure the equal distribution of sister chromatids over daughter nuclei.

Bet-hedging: also known as adaptive coin-flipping. Evolutionary risk-spreading strategy that may evolve in the face of unpredictable environmental variations, where the temporal variance in offspring fitness is minimized at the expense of their arithmetic mean fitness.

Ciliates: a eukaryotic phylum, supported by several morphological synapomorphies, e.g., somatic kinetids having a postciliary microtubular ribbon arising from triplet 9 and nuclear dimorphism. Common almost anywhere in water, with cell size varying from 10 microns to a few millimetres, mainly heterotroph but also osmotroph and parasite.

Clone: ensemble of individuals (typically cells) that share recent common ancestry through asexual reproduction.

Conflict: situation where two genetic entities (such as genomes) can each affect a joint phenotype, but they gain from moving it in opposite directions. Conflict can be ongoing, or it can be controlled, in which case we speak of potential conflict or conflict of interest [34].

Developmental switch: plasticity mechanism where alternative developmental trajectories are chosen based on information from the environment.

Generalized response: unspecific (physiological) plastic response that can lead to tolerance of a wide range of environmental conditions (for instance Heat-Shock Protein expression [10]).

Genet: A genetic individual that arises from a single zygote. May consist of multiple physiologically independent units (ramets) that are formed through vegetative growth.

Genetic assimilation: selective process by which an environmentally-induced phenotype becomes constitutively produced.

Internal Eliminated Sequence: stretch of DNA present in the micronuclear genome that is excised during macronucleus formation.

Macronucleus: the ciliate somatic nucleus. This nucleus is transcriptionally active and determines the majority of phenotypic traits. It is replaced after each sexual reproduction.

Micronucleus: the ciliate germline nucleus. Is transcriptionally silent, divides by mitosis, and undergoes meiosis preceding sexual reproduction. A mitotic sister of the micronucleus gives rise to the new macronucleus after sexual reproduction.

Nuclear dimorphism: the characteristic of having two different kinds of nuclei in a cell.

Organismality: the ensemble of properties that make a biological entity function as an organism. Such properties include cooperation and communication among its constituents, physiological integration, recognition of self and resolution of genetic conflict.

Passive phenotypic changes: any plastic change not regulated by the organism. 
Phenotypic assortment: the fixation of a haplotype (corresponding to a macronuclear chromosome) through multiple clonal divisions due to unequal chromosome segregation. Sometimes named, more adequately, allelic assortment.

Ramet: a member of a genet with a high degree of structural and physiological independence.

Reaction norm: the pattern of phenotypic trait expression of a given genotype along an environmental gradient.

Somatic mosaicism: the presence of non-heritable genetic variation within one organism.

Somatic selection: amplification of beneficial phenotypes within a pool of massively produced somatic variants through feedback with the environment. Also called developmental or epigenetic selection [8].

Superorganism: a social unit composed of individuals from the same species, manifesting a high degree of collaboration through division of labour and the presence of a specialized reproductive cast. Conflict among its constituents is reduced due to high relatedness.

Transgenerational phenotypic plasticity (intergenerational phenotypic plasticity): plastic modification resulting from the environment experienced by any ancestor, commonly referred to as parental effect when plasticity results from the environment experienced at the preceding generation.

Weismann barrier: Originally described in multicellulars as a physical distinction between immortal germ cells destined to produce gametes, and disposable somatic cells making up the body. 


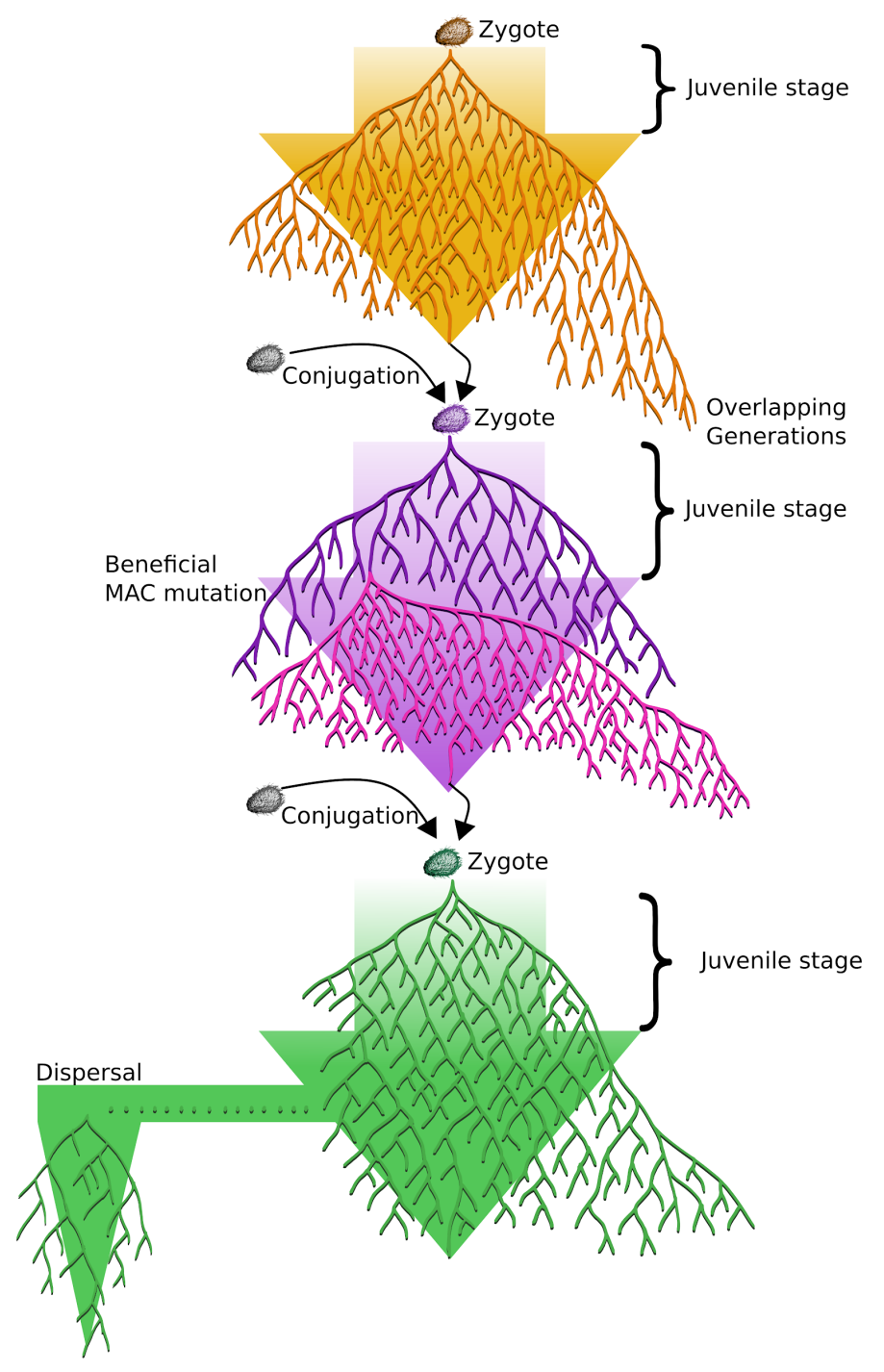

Figure 1: Schematic representation of 3 successive sexual generations in a typical ciliate

Genealogies represent dividing macronuclei, while the large arrows represent the micronuclear genotype of the entire clone. Since not all cells reproduce sexually at the same time, and different clones may be simultaneously present, generations are overlapping. For illustration purposes, only one sexual event per clone is depicted here. In the second generation, a novelty on the level of the macronucleus confers a short-term fitness benefit (pink lineage), making faster dividing cells outcompete other members of the clone that do not harbour the beneficial somatic variant. As is typically the case with phenotypic plasticity, this novelty is not passed on to the next generation. The third generation demonstrates that a clone can be spatially structured in several populations as a result of dispersal events. 


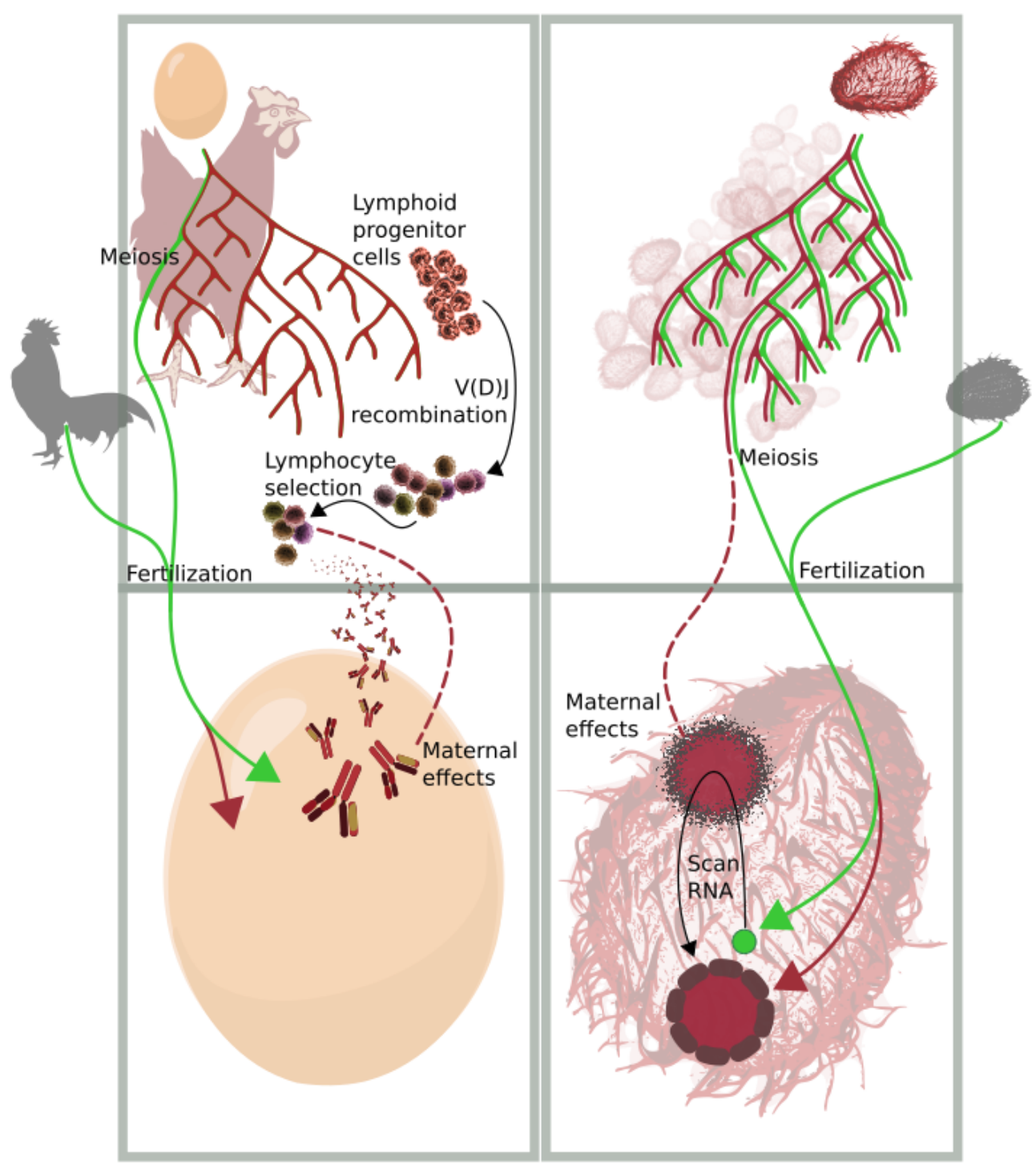

Figure 2: Comparison of phenotypic plasticity by somatic selection in a vertebrate and a ciliate

In both vertebrates (left-hand panel) and ciliates (right-hand panel), cell populations start off as a single zygote cell after fertilization. In vertebrates, the zygote gives rise to a multicellular individual with only the germline retaining its totipotency (green). The somatic cell lines (red) produce a variety of stem cells differentiating into all tissues that make up the body. One example of phenotypic plasticity by somatic selection in vertebrates is the acquired immune system. The cells giving rise to lymphoid progenitor cells undergo reshuffling of specific portions of their genome (immunoglobulin locus) through V(D)J recombination. This leads to a genetically heterogeneous population of B lymphocytes (somatic mosaicism) that receive proliferation signals depending on their match with the environment (antigen recognition). While this process generates genetic diversity at the level of the soma, the genotype of the 
individual remains unchanged, since it is sequestered in the germline. In ciliates, the cell population issued from the zygote is divisible, each cell carrying along a copy of the germline genome (green and red lines are never separated). Genetic heterogeneity is enhanced by amitosis (Fig. 3) and somatic selection acts on each macronucleus independently. In both vertebrates and ciliates, the production and elimination of unfit cells are costly and the somatic genetic adaptations are disposable. However, some non-genetic aspects of the acquired traits can be transmitted to the offspring through maternal effects, for instance by passing on antibodies in egg yolk [69] or by the structuring of the new macronucleus through scan-RNA in ciliates [70]. 


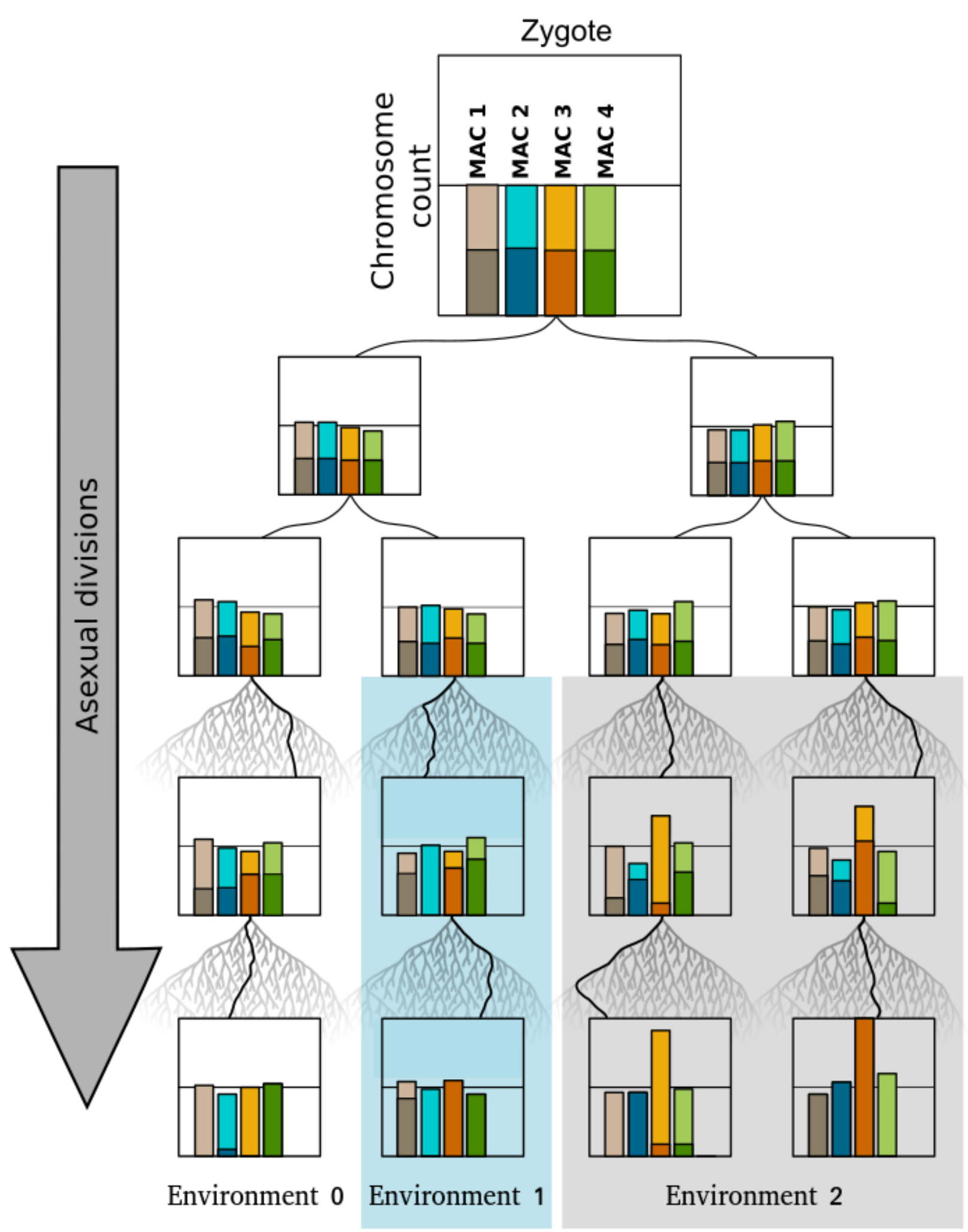




\section{Figure 3: The generation of macronuclear diversity by amitosis}

In this schematic representation of a ciliate clone, each square represents one cell. Each bar represents the counts of one macronuclear chromosome. Only 4 (out of many) macronuclear chromosomes are shown. The two colour shades within each bar represent the two parental alleles. The zygote starts off with equal copy numbers of each chromosome, with a fifty-fifty distribution of parental alleles (equal proportions of the two colour shades per bar). Since during amitosis, chromosomes are "drawn" from the pool of chromosomes like marbles from a bag, deviations will appear both in numbers of chromosomes and in ratios of parental alleles. After one or two cell divisions, small deviations from equal partitioning of chromosome numbers start to appear (bars are above or below the median line). Then, only 4 lineages are shown after multiple rounds of asexual divisions (represented by genealogical trees). They grow under different conditions. In environment 0 , which is the neutral scenario, frequencies of parental alleles deviate stochastically from equal distribution and at the end, in 3 out of 4 macronuclear chromosomes, a single parental allele has drifted to fixation (phenotypic assortment). In environment 1, the light blue allele of chromosome 2 has a selective advantage, leading to the fixation of this allele faster than expected by neutral phenotypic assortment. In environment 2 , there is a selective advantage for cells with higher copy numbers of macronuclear chromosome 3 . 


\section{BOX 1: The organismality of the ciliate clone}

Whether a group of unicellulars can be considered an organism is a long-standing discussion. Ciliates have gotten little attention in this context, despite their unique genetic configuration that makes the clone a focal unit of selection, and hence, an evolutionary individual. However, not every evolutionary individual qualifies as an organism. While authors seem to agree that nested levels of biological individuality can co-exist, criteria to define an organism are typically more restrictive $[42,63,71-73]$. Organismality is considered a derived state of a biological system resulting from feedback between natural selection and functional integration [71]. This culminates in the simultaneous concentration of evolutionary individuality (low conflict) and functional individuality (high cooperation) at one level of biological organization [42]. Though spatial contiguity is present in the majority of organisms, it is not considered a necessary condition (e.g., the eusocial insect colony) [42].

There is an important distinction between ciliates and other facultatively sexual organisms with hierarchical levels of selection. New genotypes that arise within a plant genet or a yeast clone can gain evolutionary traction and compete for their place in the gene pool. This heritable variation in fitness is a source of genetic conflict within the genet or clone $[68,74]$. In ciliates, however, somatic variation in fitness is not heritable beyond the next round of sexual reproduction due to the Weismann barrier. Therefore, individual cells do not represent a fully nested level of selection equivalent to the ramets of a plant. Much rather, the ciliate situation is reminiscent of a multicellular organism in which somatic cells benefit from the transmission of a copy of "their" genome, which does not reside in their own nucleus (Fig. 2), or of a superorganism such as a eusocial insect colony, in which individual workers serve the transmission of the genetic material of their close kin.

Organismality is also defined in terms of functional integration [5,71], or cooperation between constituents of a biological entity [42]. Little is known about cooperation in ciliates, but it has been observed that $T$. thermophila cooperates under the form of cell aggregation to exchange growth-promoting macromolecules [75,76]. Decisions to aggregate provide fitness benefits during dispersal [77] and are influenced by the presence of kin [78]. Thus, even though the individual is divisible (Fig. 1), it is capable of self/nonself discrimination, which is a property that has been highlighted as a criterion for functional individuality $[5,79]$.

Ciliates also show some degree of functional integration: depending on the age of the clone, cells are more prone to grow vegetatively or reproduce sexually. Indeed, a ciliate clone exhibits patterns of development and life-history traits that are comparable to those of a facultatively sexual multicellular with overlapping generations (Fig. 1). Each sexual generation starts off as a zygote that gives rise to a clone of vegetatively dividing cells. During the early stages of vegetative growth, processes reminiscent of embryonic development are observed in some species, with programmed macronuclear chromosome loss [80-82] and a sexually immature juvenile stage [83,84]. Senescence is also observed: after a number of asexual divisions, cells experience higher mortality and reduced fecundity, the only way to rejuvenate being sexual reproduction or autogamy. While senescence at the clone or genet level is extremely rare in clonal organisms [85], it has been observed in a wide variety of ciliates [86,87], with the notable 
exception of amicronucleated Tetrahymena lineages which, arguably, have lost the higher level of evolutionary individuality seen in other ciliates.

Many properties of ciliate biology plead in favour of viewing a ciliate clone as an organism with a shared genotype (the micronucleus), and with its generation time defined as the time between two rounds of sexual reproduction. This viewpoint is further corroborated by the fact that micronuclear mutation rates only fall within the range of known eukaryotic mutation rates per generation if we consider the sexual generation time $[88,89]$. The discussion about the extent of organismality of the ciliate clone needs further input from experiments and observations, especially about functional integration and cooperation. Given that organismality is a continuous $[42,68,71]$ and context-dependent [63] quantity, it may well be that different ciliate species take a different place in this spectrum. 


\section{References}

1 Qu, Z. et al. (2020) Aquatic food webs in deep temperate lakes: key species establish through their autecological versatility. Mol. Ecol. 30, 1053-1071

2 Rzeszutek, I. et al. (2020) Programmed genome rearrangements in ciliates. Cell. Mol. Life Sci. 77, 4615-4629

3 Lewontin, R.C. (1970) The Units of Selection. Annu. Rev. Ecol. Syst. 1, 1-18

4 Wilson, J. (1999) Biological Individuality: The Identity and Persistence of Living Entities, Cambridge University Press

5 Pradeu, T. (2016) Organisms or biological individuals? Combining physiological and evolutionary individuality. Biol. Philos. 31, 797-817

6 Uller, T. (2008) Developmental plasticity and the evolution of parental effects. Trends Ecol. Evol. 23, 432-438

7 Sommer, R.J. (2020) Phenotypic Plasticity: From Theory and Genetics to Current and Future Challenges. Genetics 215, 1-13

8 West-Eberhard, M. (2003) Developmental Plasticity And Evolution, Oxford University Press

9 Fusco, G. and Minelli, A. (2010) Phenotypic plasticity in development and evolution: facts and concepts. Philos. Trans. R. Soc. B Biol. Sci. 365, 547-556

10 Snell-Rood, E.C. et al. (2018) Mechanisms of Plastic Rescue in Novel Environments. Annu. Rev. Ecol. Evol. Syst. 49, 331-354

11 Jacob, F. and Monod, J. (1961) Genetic regulatory mechanisms in the synthesis of proteins. J. Mol. Biol. 3, 318-356

12 Dubravcic, D. et al. (2014) An evolutionarily significant unicellular strategy in response to starvation in Dictyostelium social amoebae. F1000Research 3: 133

13 Perry, B.W. et al. (2018) Evolution: Plasticity versus Selection, or Plasticity and Selection? Curr. Biol. 28, R1104-R1106

14 Ghalambor, C.K. et al. (2007) Adaptive Versus Non-Adaptive Phenotypic Plasticity and the Potential for Contemporary Adaptation in New Environments. Funct. Ecol. 21, 394-407

15 Beaumont, H.J.E. et al. (2009) Experimental evolution of bet hedging. Nature 462, 90-93

16 de Jong, I.G. et al. (2011) Bet hedging or not? A guide to proper classification of microbial survival strategies. BioEssays 33, 215-223

17 Bruijning, M. et al. (2019) The Evolution of Variance Control. Trends Ecol. Evol. 35, 22-33

18 Ackermann, M. (2015) A functional perspective on phenotypic heterogeneity in microorganisms. Nat. Rev. Microbiol. 13, 497-508

19 Rotem, E. et al. (2010) Regulation of phenotypic variability by a threshold-based mechanism underlies bacterial persistence. Proc. Natl. Acad. Sci. 107, 12541-12546

20 Chaturvedi, S. et al. (2020) A molecular mechanism for probabilistic bet hedging and its role in viral latency. Proc. Natl. Acad. Sci. 117, 17240-17248

21 Sadeh, A. et al. (2009) Plastic Bet-Hedging in an Amphicarpic Annual: An Integrated Strategy Under Variable Conditions. Evol. Ecol. 23, 373-388

22 Snell-Rood, E.C. (2012) Selective Processes in Development: Implications for the Costs and Benefits of Phenotypic Plasticity. Integr. Comp. Biol. 52, 31-42

23 Fernando, C. et al. (2012) Selectionist and Evolutionary Approaches to Brain Function: A Critical Appraisal. Front. Comput. Neurosci. 6:24

24 Maurer-Alcalá, X.X. et al. (2018) Twisted Tales: Insights into Genome Diversity of Ciliates Using Single-Cell 'Omics. Genome Biol. Evol. 10, 1927-1939 
25 Prescott, D.M. (1994) The DNA of ciliated protozoa. Microbiol. Rev. 58, 233-267

26 Doerder, F.P. et al. (1992) Rate of phenotypic assortment in Tetrahymena thermophila. Dev. Genet. 13, 126-132

27 Larson, D.D. et al. (1991) Copy Number Control in the Tetrahymena Macronuclear Genome. J. Protozool. 38, 258-263

28 Spring, K.J. et al. (2013) Chromosome copy number variation and control in the ciliate Chilodonella uncinata. PloS One 8, e56413

29 Bellec, L. and Katz, L.A. (2012) Analyses of chromosome copy number and expression level of four genes in the ciliate Chilodonella uncinata reveal a complex pattern that suggests epigenetic regulation. Gene 504, 303-308

$30 \mathrm{Xu}, \mathrm{K}$. et al. (2012) Copy number variations of 11 macronuclear chromosomes and their gene expression in Oxytricha trifallax. Gene 505, 75-80

31 Cheng, Y.-H. et al. (2020) Genome plasticity in Paramecium bursaria revealed by population genomics. BMC Biol. 18, 180

32 de Francisco, P. et al. (2018) Genome plasticity in response to stress in Tetrahymena thermophila: selective and reversible chromosome amplification and paralogous expansion of metallothionein genes. Environ. Microbiol. 20, 2410-2421

33 McLaughlin, R.N. and Malik, H.S. (2017) Genetic conflicts: the usual suspects and beyond. J. Exp. Biol. 220, 6-17

34 Queller, D.C. and Strassmann, J.E. (2018) Evolutionary Conflict. Annu. Rev. Ecol. Evol. Syst. 49, 73-93

35 Gardner, A. and Úbeda, F. (2017) The meaning of intragenomic conflict. Nat. Ecol. Evol. 1, 1807-1815

36 Taylor, D.R. et al. (2002) Conflicting levels of selection in the accumulation of mitochondrial defects in Saccharomyces cerevisiae. Proc. Natl. Acad. Sci. 99, 3690-3694

37 Crespi, B. and Summers, K. (2005) Evolutionary biology of cancer. Trends Ecol. Evol. 20, 545-552

38 Wenseleers, T. et al. (2020) Conflicts of Interest Within Colonies. In Encyclopedia of Social Insects (Starr, C. K., ed), pp. 1-15, Springer International Publishing

39 Birky, C.W. (1995) Uniparental inheritance of mitochondrial and chloroplast genes: mechanisms and evolution. Proc. Natl. Acad. Sci. 92, 11331-11338

40 Doerder, F.P. (1996) Nuclear wars: The relationship between the micronucleus and the macronucleus in ciliate protists. Eur. J. Protistol. 32, 14-18

41 Maurer-Alcalá, X.X. and Nowacki, M. (2019) Evolutionary origins and impacts of genome architecture in ciliates. Ann. N. Y. Acad. Sci. 1447, 110-118

42 Queller, D.C. and Strassmann, J.E. (2009) Beyond society: the evolution of organismality. Philos. Trans. R. Soc. Lond. B Biol. Sci. 364, 3143-3155

43 Roper, M. et al. (2011) Nuclear and Genome Dynamics in Multinucleate Ascomycete Fungi. Curr. Biol. 21, R786-R793

44 Doerder, F.P. (2014) Abandoning sex: multiple origins of asexuality in the ciliate Tetrahymena. BMC Evol. Biol. 14, 112

45 Schurko, A.M. et al. (2009) Signs of sex: what we know and how we know it. Trends Ecol. Evol. 24, 208-217

46 Dawson, J.A. (1919) An experimental study of an amicronucleate Oxytricha. J. Exp. Zool. 29, 473-513

47 Yan, Y. et al. (2017) Unusual features of non-dividing somatic macronuclei in the ciliate class Karyorelictea. Eur. J. Protistol. 61, 399-408

48 Michod, R.E. et al. (2003) Cooperation and conflict in the evolution of individuality: IV. 
Conflict mediation and evolvability in Volvox carteri. Biosystems 69, 95-114

49 Hammerschmidt, K. et al. (2014) Life cycles, fitness decoupling and the evolution of multicellularity. Nature 515, 75-79

50 Stencel, A. and Suárez, J. (2021) Do Somatic Cells Really Sacrifice Themselves? Why an Appeal to Coercion May be a Helpful Strategy in Explaining the Evolution of Multicellularity. Biol. Theory 16, 102 - 113

51 Raikov, I.B. (1994) The diversity of forms of mitosis in protozoa: a comparative review. Eur. J. Protistol. 30, 253-269

52 Yerlici, V.T. and Landweber, L.F. (2014) Programmed genome rearrangements in the ciliate Oxytricha. Microbiol. Spectr. 2: 6

53 Klobutcher, L.A. and Herrick, G. (1997) Developmental genome reorganization in ciliated protozoa: the transposon link. Prog. Nucleic Acid Res. Mol. Biol. 56, 1-62

54 Yao, M.-C. and Chao, J.-L. (2005) RNA-guided DNA deletion in Tetrahymena: an RNAi-based mechanism for programmed genome rearrangements. Annu. Rev. Genet. 39, 537-559

55 Vogt, A. et al. (2013) Transposon Domestication versus Mutualism in Ciliate Genome Rearrangements. PLOS Genet. 9 (8), e1003659

56 Vitali, V. et al. (2019) Environmentally induced plasticity of programmed DNA elimination boosts somatic variability in Paramecium tetraurelia. Genome Res. 29, 1693-1704

57 Hai, B. and Gorovsky, M.A. (1997) Germ-line knockout heterokaryons of an essential a-tubulin gene enable high-frequency gene replacement and a test of gene transfer from somatic to germ-line nuclei in Tetrahymena thermophila. Proc. Natl. Acad. Sci. 94, 1310-1315

58 Danchin, É. et al. (2011) Beyond DNA: integrating inclusive inheritance into an extended theory of evolution. Nat. Rev. Genet. 12, 475-486

59 Danchin, É. (2013) Avatars of information: towards an inclusive evolutionary synthesis. Trends Ecol. Evol. 28, 351-358

60 Heyse, G. et al. (2010) RNA-dependent control of gene amplification. Proc. Natl. Acad. Sci. $107,22134-22139$

61 Nowacki, M. et al. (2010) RNA-mediated epigenetic regulation of DNA copy number. Proc. Natl. Acad. Sci. 107, 22140-22144

62 Chalker, D.L. and Yao, M.C. (1996) Non-Mendelian, heritable blocks to DNA rearrangement are induced by loading the somatic nucleus of Tetrahymena thermophila with germ line-limited DNA. Mol. Cell. Biol. 16, 3658-3667

63 Díaz-Muñoz, S.L. et al. (2016) Contextual organismality: Beyond pattern to process in the emergence of organisms. Evolution 70, 2669-2677

64 Aanen, D.K. (2019) Germline Evolution: Sequestered Cells or Immortal Strands? Curr. Biol. 29, R799-R801

65 Aanen Duur K. and Debets Alfons J. M. (2019) Mutation-rate plasticity and the germline of unicellular organisms. Proc. R. Soc. B Biol. Sci. 286, 20190128

66 Lanfear, R. (2018) Do plants have a segregated germline? PLOS Biol. 16, e2005439

67 Plomion, C. et al. (2018) Oak genome reveals facets of long lifespan. Nat. Plants 4, 440-452

68 Clarke, E. (2012) Plant individuality: a solution to the demographer's dilemma. Biol. Philos. 27, 321-361

69 Gasparini, J. et al. (2006) Dynamics of anti-Borrelia antibodies in Black-legged Kittiwake (Rissa tridactyla) chicks suggest a maternal educational effect. Can. J. Zool. 84, 623-627

70 Chalker, D.L. and Yao, M.-C. (2011) DNA Elimination in Ciliates: Transposon Domestication and Genome Surveillance. Annu. Rev. Genet. 45, 227-246 
71 Pepper, J.W. and Herron, M.D. (2008) Does biology need an organism concept? Biol. Rev. Camb. Philos. Soc. 83, 621-627

72 West, S.A. and Kiers, E.T. (2009) Evolution: What Is an Organism? Curr. Biol. 19, R1080-R1082

73 Wilson, J.A. (2000) Ontological Butchery: Organism Concepts and Biological Generalizations. Philos. Sci. 67, S301-S311

74 Sober, E. and Wilson, D.S. (1994) A Critical Review of Philosophical Work on the Units of Selection Problem. Philos. Sci. 61, 534-555

75 Christensen, S.T. et al. (1995) Mechanisms controlling death, survival and proliferation in a model unicellular eukaryote Tetrahymena thermophila. Cell Death Differ. 2, 301-308

76 Jacob, S. et al. (2016) Social Information in Cooperation and Dispersal in Tetrahymena. In Biocommunication of Ciliates (Witzany, G. and Nowacki, M., eds), pp. 235-252, Springer International Publishing

77 Jacob, S. et al. (2016) Cooperation-mediated plasticity in dispersal and colonization. Evolution 70, 2336-2345

78 Chaine, A.S. et al. (2009) Kin-based recognition and social aggregation in a ciliate. Evolution 64, 1290-1300

79 Pradeu, T. (2019) Immunology and individuality. eLife 8, e47384

80 Hamilton, E.P. et al. (2016) Structure of the germline genome of Tetrahymena thermophila and relationship to the massively rearranged somatic genome. eLife 5, e19090

81 Clay, D.M. et al. (2019) Programmed Chromosome Deletion in the Ciliate Oxytricha trifallax. G3 9, 3105-3118

82 Lin, C.-Y.G. et al. (2016) Programmed Minichromosome Elimination as a Mechanism for Somatic Genome Reduction in Tetrahymena thermophila. PLoS Genet. 12, e1006403

83 Dini, F. and Nyberg, D. (1992) Development of sexual maturity in the ciliate Euplotes crassus: Sources of variation in the timing of maturity. Dev. Genet. 13, 41-46

84 Rogers, M.B. and Karrer, K.M. (1985) Adolescence in Tetrahymena thermophila. Proc. Natl. Acad. Sci. 82, 436-439

85 Shefferson, R.P. et al., eds. (2017) The Evolution of Senescence in the Tree of Life, Cambridge University Press.

86 Aufderheide, K.J. (1984) Clonal aging in Paramecium tetraurelia, absence of evidence for a cytoplasmic factor. Mech. Ageing Dev. 28, 57-66

87 Adl, S.M. and Berger, J.D. (2000) Timing of Life Cycle Morphogenesis in Synchronous Samples of Sterkiella histriomuscorum. II. The Sexual Pathway. J. Eukaryot. Microbiol. 47, 443-449

88 Sung, W. et al. (2012) Extraordinary genome stability in the ciliate Paramecium tetraurelia. Proc. Natl. Acad. Sci. 109, 19339-19344

89 Long, H. et al. (2016) Low Base-Substitution Mutation Rate in the Germline Genome of the Ciliate Tetrahymena thermophila. Genome Biol. Evol. 8, 3629-3639 